\title{
3-Phase Current Reconstruction of Permanent Magnet Synchronous Motor Drives Using a Single DC-Link Current Sensor
}

\author{
Cheng-Yu Hsieh, Wen-Chun Chi, Ming-Yang Cheng, ${ }^{*}$ \\ Wei-Liang Kuo, and Wei-Leng Wu \\ Department of Electrical Engineering, National Cheng Kung University, Tainan, Taiwan \\ (Received February 25, 2015; accepted February 26, 2016)
}

Keywords: 3-phase current reconstruction, SVPWM, PMSM

In the application of conventional 3-phase current reconstruction methods using a single DC-link current shunt, the self-switch configuration of space vector pulse width modulation (SVPWM) may cause a problem of insufficient measuring time in certain regions. To cope with this problem, in this paper, we propose a switching strategy that exploits the advantages of SVPWM, the measurement vector insertion method (MVIM), and the pulse-width modulation method without using the null switching states method (NSVM). In the proposed switching strategy, the MVIM is employed in the low-modulation-ratio region and the sector boundary region of the middle-modulation-ratio region, while the NSVM is adopted in the sector boundary region of the high-modulation-ratio region. In addition, we used Matlab/Simulink in this study to construct a simulated environment to conduct an in-depth study of the differences among various current construction methods as well as the size of the immeasurable region.

\section{Introduction}

The 3-phase current information of the permanent magnet synchronous motor (PMSM) plays a crucial role in closed-loop control as well as in overcurrent protection, DC-link current short protection, and ground fault protection. The safety of PMSM and its drive can be ensured if one can use 3-phase current feedback information effectively. Common approaches for measuring 3-phase currents use two current sensors in series with phase winding. Although the approach is easy to implement, it is not cost-effective. Moreover, to ensure accuracy in phase current reconstruction, parameters such as current magnification, DC offset, and filter cutoff frequency for the two current sensors must be in good agreement. Otherwise, when reconstructing the current of the third phase, differences in parameter values may lead to errors in the reconstructed phase current so that the output ripple of the current loop may increase. (1) A possible approach to coping with this problem is to use a single DC-link current sensor to retrieve phase current information. Since only one current sensor is used, the cost is lower and the problem of deviation in parameter values for different current sensors does not exist.

Suppose that the 3-phase current reconstruction technique is implemented on the basis of a single DC-link current sensor and space vector pulse width modulation (SVPWM). When the

"Corresponding author: e-mail: mycheng@mail.ncku.edu.tw 
voltage space vector is located in the sector boundary region of the voltage vector, or when the modulation ratio of the reference voltage vector is too small, the component of the reference voltage vector will be too small. As a result, the power switching conduction time is so short that the time duration of the voltage vector is shorter than the minimum time needed for measuring the phase current. Since in this region the phase current cannot be correctly measured, ${ }^{(2)}$ this leads to error in the reconstruction of the 3-phase current. To solve these problems, many approaches for dealing with the problem of 3-phase current reconstruction using a single DC-link current sensor have been proposed. In this paper, we describe an in-depth study on several existing modified SVPWMbased reconstruction methods. The advantages and drawbacks of these existing approaches are analyzed. On the basis of these results, a switching strategy has been developed, which exhibits the advantages of SVPWM, a pulse width modulation method without using the null switching states method (NSVM), ${ }^{(5-7)}$ and the measurement vector insertion method (MVIM). ${ }^{(8)}$ In particular, MVIM is employed in the low-modulation-ratio region and the sector boundary region of the middle-modulation-ratio region, while the NSVM is used in the sector boundary region of the highmodulation-ratio region.

\section{Methods}

\subsection{Phase current reconstruction limitation of SVPWM}

Assume that the motor drive control of a PMSM is implemented using SVPWM. If the space vector of the inverter is an effective voltage vector, one can determine to which phase the current signal measured by the DC-link current sensor belongs. By sampling the resultant reference voltage vector of two effective vectors during one SVPWM period and applying the principle of a balanced 3-phase system, one can reconstruct the 3-phase currents based on the phase current measured by the DC-link current sensor. To correctly reconstruct the 3-phase currents, the reference voltage vector of the inverter must satisfy the following two inequalities: ${ }^{(9)}$

$$
\begin{gathered}
T_{\text {acq }} \leq T_{1}=T_{\mathrm{z}} \cdot m \cdot \frac{\sin \left(\pi / 3-\theta_{\mathrm{e}}\right)}{\sin (\pi / 3)}, \\
T_{\text {acq }} \leq T_{2}=T_{\mathrm{z}} \cdot m \cdot \frac{\sin \left(\theta_{\mathrm{e}}\right)}{\sin (\pi / 3)},
\end{gathered}
$$

where $T_{\text {acq }}$ is the minimum detection time of the phase current, and $T_{1}$ and $T_{2}$ are the times needed for the conduction of the space vectors $\vec{V}_{1}$ and $\vec{V}_{2}$, respectively. The value of $T_{\mathrm{z}}$ is half of the switching time for a symmetric SVPWM. If either Eqs. (1) or (2) is not satisfied, the reconstruction of the 3-phase currents may be in error. Figure 1 illustrates the region of the reference voltage vector that can reconstruct the 3-phase currents under SVPWM. The analysis of the region for which the reference voltage vector is located is provided in the following:

In region (A) of Fig. 1, one can reconstruct the 3-phase currents correctly. In this region, both the time duration of the effective vectors of $T_{1}$ and $T_{2}$ are larger than that of $T_{\text {acq. }}$. If the reference voltage vector is located in this region, one can reconstruct the 3-phase currents without performing any change with respect to the output signal of SVPWM.

In region (B) of Fig. 1, one cannot reconstruct the 3-phase currents correctly. This region is commonly called the sector boundary region. ${ }^{(3,4)}$ In this region, only one of the time durations of 


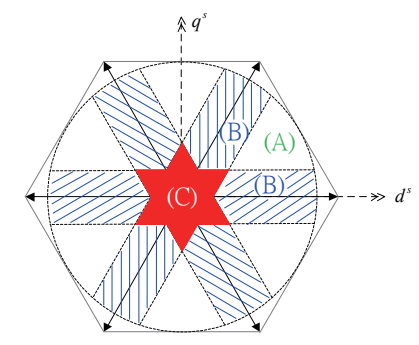

Fig. 1. (Color online) Illustrative diagram of region of 3-phase current reconstruction using SVPWM.

the effective vectors of $T_{1}$ and $T_{2}$ is larger than $T_{\text {acq. }}$. As a result, only the current information from one phase of SVPWM is available. That is, the reconstruction of the 3-phase currents is impossible.

In region (C), one cannot reconstruct the 3-phase currents correctly. This region is commonly referred to as the low-modulation-ratio region, ${ }^{(3,4)}$ in which both the time durations of the effective vectors of $T_{1}$ and $T_{2}$ are smaller than $T_{\text {acq }}$. Therefore, no phase current information can be acquired during the SVPWM period.

\subsection{Modulation method without using NSVM}

As shown in Fig. 1, regions (B) and (C) are the regions for which the 3-phase current reconstruction technique using a single DC-link current sensor fails (defined as the immeasurable region in this paper). To reduce the size of regions (B) and (C), several previous studies have proposed a modified approach. That is, when the reference voltage vector is located in the immeasurable region, the output signal of SVPWM is provided by NSVM..$^{(5-7)}$ Moreover, phase current detection is carried out with respect to the output signal provided by NSVM. As shown in Fig. 2, in the NVSM approach, $V_{0}$ and $V_{7}$ of SVPWM are replaced by $V_{\mathrm{M}}$ and $V_{\overline{\mathrm{M}}}$, respectively.

\section{$2.3 \quad$ MVIM}

In Ref. 8, the MVIM is proposed to overcome the difficulty in which the NSVM may lead to large current harmonics in the low-modulation-ratio region. In the MVIM, if the reference voltage vector is located in the immeasurable region of the SVPWM, then a measurement vector is inserted in the output signals, and the current detection is carried out at this measurement vector as shown in Fig. 3. When the switching frequency is high, the total output power of these three effective vectors is zero for the motor itself. The time duration $T_{\mathrm{mv}}$ for these three effective vectors is decided by the minimum detection time of the phase current $T_{\text {acq. }}$.

\section{Results}

To conduct a complete and extensive analysis of different 3-phase current reconstruction methods, we used Matlab/Simulink to construct a simulated environment to investigate the differences among various current construction methods as well as the size of the immeasurable region and current harmonics. Figure 4 shows the simulation results for the case of the 0.95 (p.u.) reference voltage vector, while Fig. 5 shows the simulated results for the case of the 0.2 (p.u.) reference voltage vector. The results indicate that the NVSM can indeed reduce the size of the immeasurable region in the sector boundary region and result in small current harmonics in the 


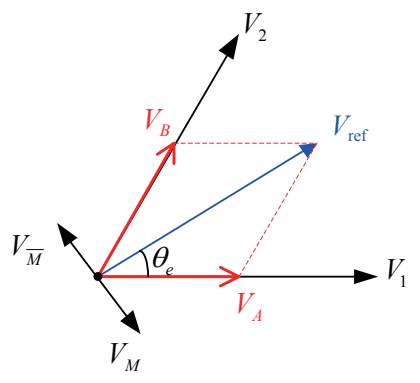

Fig. 2. (Color online) Illustrative diagram of NSVM in the voltage vector space.

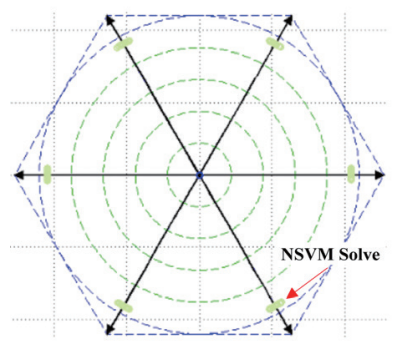

(a)

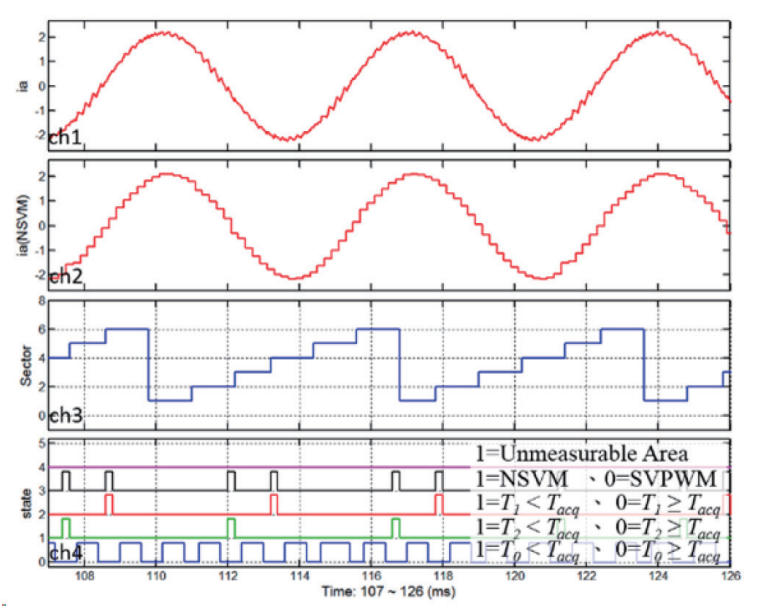

(b)

Fig. 4. (Color online) Simulated results of NSVM in the high-modulation-ratio region $\left[F_{\mathrm{pwm}}=5 \mathrm{kHz}\right.$ and $V_{\text {ref }}=0.95$ (p.u.)]. (a) Immeasurable region and (b) simulated waveform.

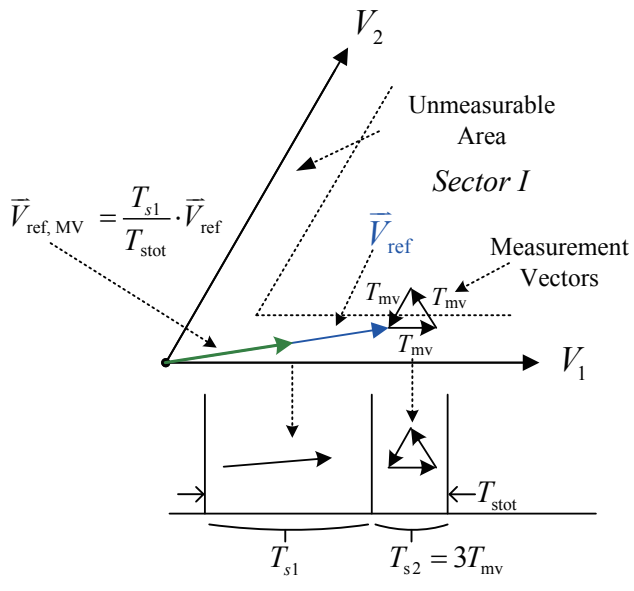

Fig. 3. (Color online) Illustrative diagram of MVIM in the voltage vector space.

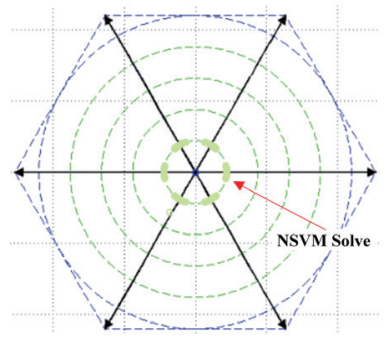

(a)

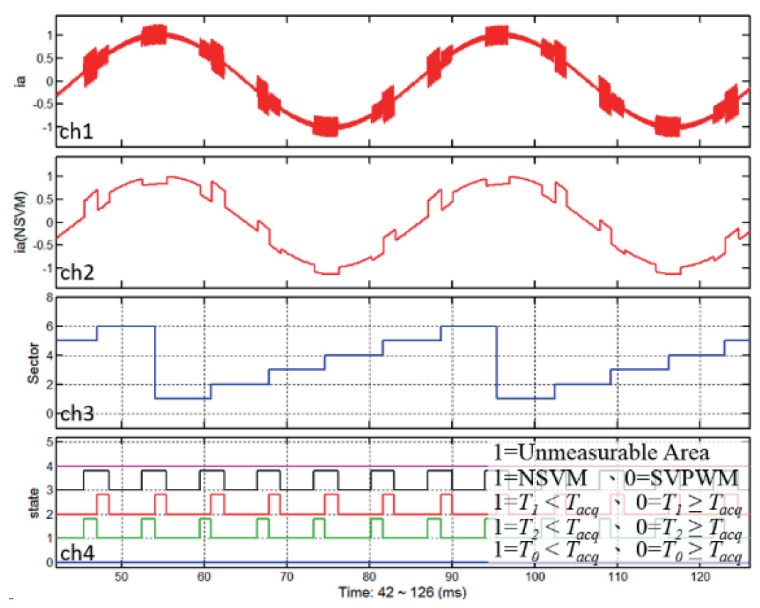

(b)

Fig. 5. (Color online) Simulated results of NSVM in the low-modulation-ratio region $\left[F_{\mathrm{pwm}}=5 \mathrm{kHz}\right.$ and $V_{\text {ref }}=0.2$ (p.u.)]. (a) Immeasurable region and (b) simulated waveform. 
high-modulation-ratio region. However, the NVSM results in large current harmonics in the lowmodulation-ratio region. Therefore, the NVSM is not suitable for the low-modulation-ratio region. In contrast, Figs. 6 and 7 show the simulated results of MVIM in the middle- and low-modulationratio regions, respectively. These results reveal that the MVIM can be used to reconstruct the 3-phase currents in the low-modulation-ratio region, resulting in small current harmonics. Consequently, the MVIM can be adopted in the low-modulation-ratio region to reconstruct the 3-phase currents.

\section{Discussion}

Table 1 lists the advantages and drawbacks of different 3-phase current reconstruction methods such as SVPWM, NSVM, and MVIM. Clearly, each method has its own suitable modulation ratio region. To reduce the size of the immeasurable region, we propose a switching strategy that exploits the advantages of the three 3-phase current reconstruction methods. In particular, by considering the advantages and drawbacks of the three methods, the proposed approach adopts different current reconstruction methods in different regions of the space vector as listed in Table 2 and shown in Fig. 8. For example, if the reference voltage vector is located in region (A), then the original SVPWM-based method is adopted to reconstruct the 3-phase current. In contrast, if the

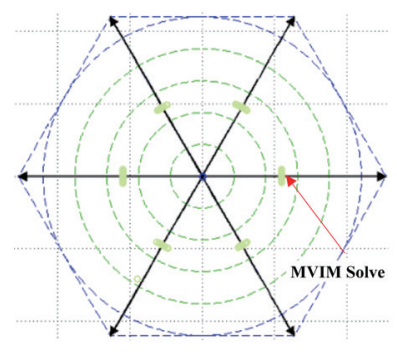

(a)

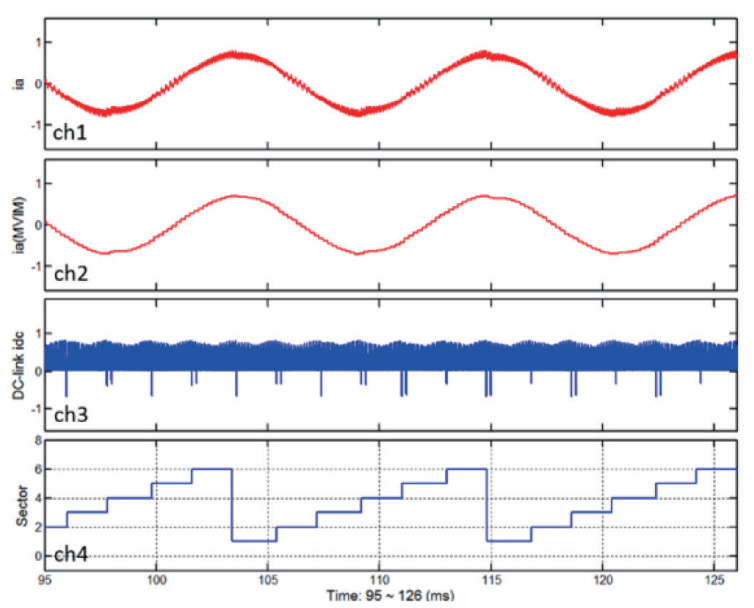

(b)

Fig. 6. (Color online) Simulated results of MVIM in the middle-modulation-ratio region $\left[F_{\mathrm{pwm}}=5 \mathrm{kHz}\right.$ and $V_{\text {ref }}=0.5$ (p.u.)]. (a) Immeasurable region and (b) simulated waveform.

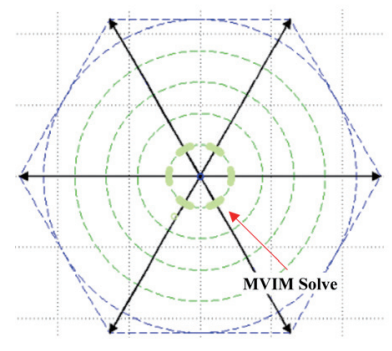

(a)

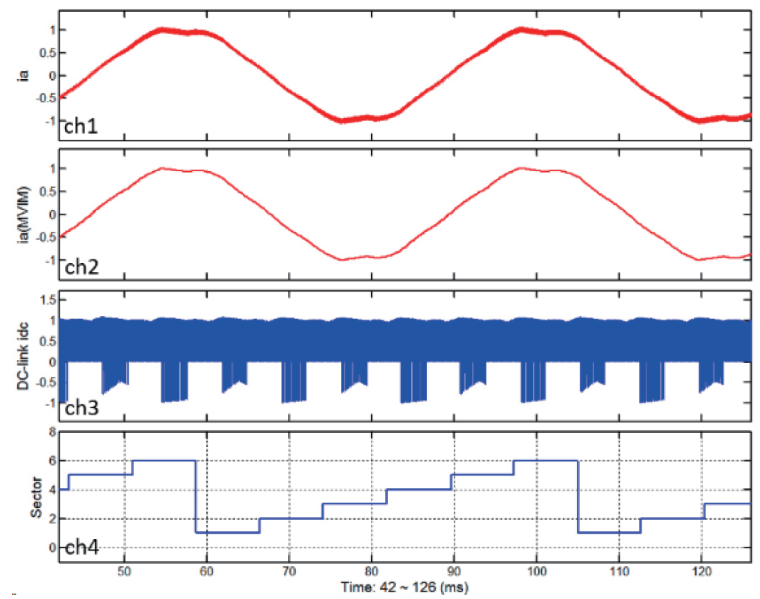

(b)

Fig. 7. (Color online) Simulated results of MVIM in the low-modulation-ratio region $\left[F_{\mathrm{pwm}}=5 \mathrm{kHz}\right.$ and $V_{\text {ref }}=0.2$ (p.u.)]. (a) Immeasurable region and (b) simulated waveform. 
Table 1

Drawbacks and advantages of three 3-phase current reconstruction methods using a single DC-link current sensor.

\begin{tabular}{lll}
\hline Methods & Comparison & Description \\
\cline { 2 - 3 } SVPWM & Advantage & Lowest current harmonics. \\
\cline { 2 - 3 } Drawback & $\begin{array}{l}\text { Immeasurable regions consist of the sector boundary region of the middle- and } \\
\text { high-modulation-ratio regions and the low modulation-ratio region. }\end{array}$ \\
\hline \multirow{2}{*}{ MVVM } & Advantage & Small immeasurable regions in the middle- and high-modulation-ratio regions. \\
\cline { 2 - 3 } & Drawback & Large current harmonics in the low-modulation-ratio region. \\
\cline { 2 - 3 } & Advantage & Can reconstruct 3-phase current in the middle- and high-modulation-ratio regions. \\
\hline
\end{tabular}

Table 2

The proposed switching strategy for 3-phase current reconstruction.

\begin{tabular}{lc}
\hline Region & Adopted methods \\
\hline (A) & SVPWM \\
(B) & NSVM or MVIM \\
(C) & MVIM \\
(D) & NSVM \\
\hline
\end{tabular}

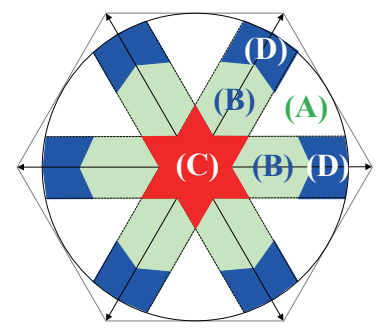

Fig. 8. (Color online) Illustrative diagram of the proposed switching strategy.

reference voltage vector is located in regions (C) or (D), then MVIM (or NSVM) is adopted. If the reference voltage vector is located in region (B), either MVIM or NSVM can be used.

\section{Conclusions}

In this study, we constructed a simulated environment based on Matlab/Simulink to investigate the advantages and drawbacks of several existing 3-phase current construction methods. On the basis of the analysis of the Matlab/Simulink simulation results, in this study, we proposed a switching strategy that exploits the advantages of three 3-phase current reconstruction methods. In particular, by analyzing the advantages and drawbacks of the 3-phase current reconstruction methods, the proposed switching strategy can be used to determine a suitable 3-phase current reconstruction method for different regions of the space vector to provide a wider 3-phase current reconstruction range than other existing methods.

\section{References}

1 H. S. Jung, S. H. Hwang, J. M. Kim, C. U. Kim, and C. Choi: IEEE Trans. Ind. Appl. 42 (2006) 1249.

2 J. Moynihan, R. Kavanagh, M. Egan, and J. Murphy: Proc. Conf. Power Electron. Appl. 1992 (IEEE, New York, 1992) pp. 641-641.

3 W. C. Lee, T. K. Lee, and D. S. Hyun: IEEE Trans. Ind. Electron. 48 (2001) 491.

4 Y. Gu, F. Ni, D. Yang, and H. Liu: IEEE Trans. Ind. Electron. 58 (2011) 5186.

5 Y. S. Lai: Proc. Conf. IEEE Power Eng. Soc. 1999 (IEEE, New York, 1999) pp. 35-40.

6 Y. S. Lai and F. S. Shyu: IEEE Trans. Ind. Appl. 40 (2004) 1605.

7 Y. S. Lai, P. S. Chen, H. K. Lee, and J. Chou: IEEE Trans. Ind. Appl. 40 (2004) 1613.

8 H. Kim and T. M. Jahns: IEEE Trans. Power Electron. 21 (2006) 1413.

9 Y. V. S. Reddy, T. B. Reddy, and M. V. Kumar: Proc. Int. Conf. Power Electron. Drives Energy Syst. 2006 (IEEE, New York, 2006) pp. 1-5. 